\title{
Small-bowel perforation in a patient with \\ Roux-en-Y anatomy for Peutz-Jeghers polyps after spiral enteroscopic investigation
}

Spiral enteroscopy is a new technique for diagnosis and treatment of small-bowel disease. In July 2009, a 19-year-old woman with Peutz-Jeghers syndrome was sent to our hospital for screening and treatment. The patient had undergone segmental resection of the distal jejunum, 4 years previously, for acute intestinal obstruction due to large Peutz-Jeghers polyps.

After informed consent had been obtained, we chose antegrade spiral enteroscopy for small-bowel investigation. The procedure was performed under monitored anesthesia. A standard Fujinon EN-450P5 enteroscope with an Endo-Ease Discovery $\mathrm{SB}$ overtube was used. The insertion depth of the enteroscope was about $220 \mathrm{~cm}$ beyond the ligament of Treitz. The duration of the procedure was $44 \mathrm{~min}$.

During the investigation, a normal Rouxen-Y anastomotic stoma was found at about $200 \mathrm{~cm}$ beyond the ligament of Treitz ( $\bullet$ Fig. 1), and eight Ila-type hyperplastic polyps of size $3-8 \mathrm{~mm}$ were found nearby. Argon plasma coagulation (APC) was used for treatment of these polyps.

The procedure of investigation and treatment was successful, and no bleeding occurred. However, the patient experienced severe abdominal pain after $2 \mathrm{~h}$. The abdominal computed tomography (CT) scan showed pneumoperitoneum. During emergent surgery, a perforation of $15 \mathrm{~mm}$ was seen at the anastomotic stoma, and intraperitoneal adhesion was severe. The patient was clinically stable after repair of the perforation, and was discharged 5 days later.

Diagnostic double-balloon enteroscopy (DBE) has an overall complication rate of $1.7 \%$ (perforation $0.3 \%$, pancreatitis $0.3 \%$, bleeding $0.8 \%$ ). Therapeutic DBE has a relatively high complication rate of $4.3 \%$

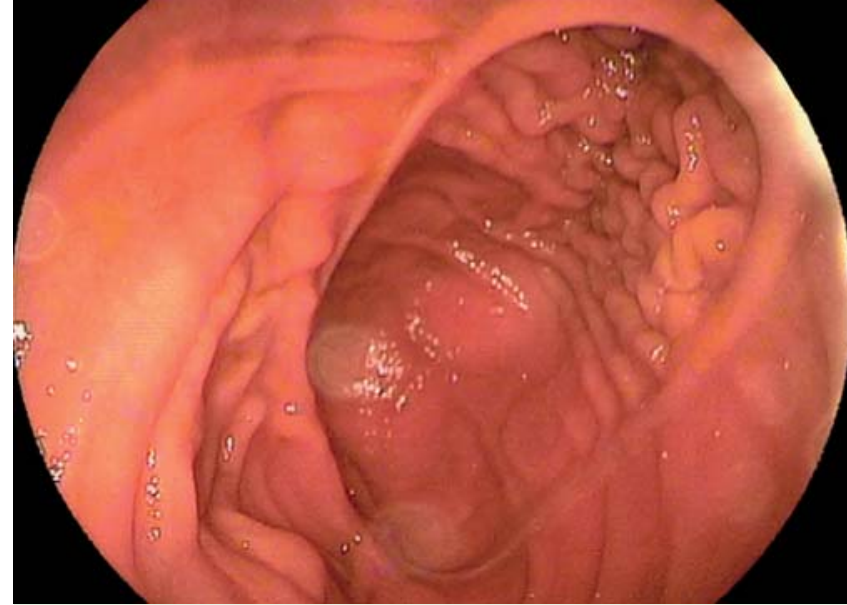

Fig. 1 Endoscopic view, showing the anastomotic stoma in the distal jejunum.

(APC perforation $1.2 \%$, dilation perforation $2.9 \%$ ) [1]. In patients with small-bowel anastomoses, perforations occur easily during diagnostic DBE procedures [2]. The rate of perforation after spiral enteroscopy is reported to be $0.34 \%$ [3]. Spiral enteroscopy has been successfully performed in patients with surgically altered anatomies [4], with no reports of perforation until now. In this case, perforation was found to have occurred because of prior surgery not therapeutic procedures. Avoiding excessive tension may help to limit complications including perforation.

\section{Endoscopy_UCTN_Code_CPL_1AI_2AD}

\section{Competing interests: None}

X.-B. Li ${ }^{1,2}$, L.-Y. Gu' ${ }^{1}$, Y. Song ${ }^{1}$, Y.-J. Gao ${ }^{1}$, Z.-Z. $\mathrm{Ge}^{1,2}$

1 Department of Gastroenterology,

Shanghai Renji Hospital, Shanghai Jiaotong University School of Medicine, Shanghai, China

2 Shanghai Institute of Digestive Disease, Shanghai, China

\section{References}

1 Mensink PB, Haringsma J, Kucharzik T et al. Complications of double balloon enteroscopy: a multicenter survey. Endoscopy 2007; 39: $613-615$

2 Semrad CE. Small-bowel enteroscopy: territory conquered, future horizons. Curr Opin Gastroenterol 2009; 25: 110-115

3 Akerman PA, Cantero D. Severe complications of spiral enteroscopy in the first 1750 patients. Gastrointest Endosc 2009; 69: AB127

4 Akerman PA, Agrawal D, Chen W et al. Spiral enteroscopy: a novel method of enteroscopy by using the Endo-Ease Discovery SB overtube and a pediatric colonoscope. Gastrointest Endosc 2009; 69: 327 - 332

Bibliography

Dol $10.1055 / \mathrm{s}-0030-1255897$

Endoscopy 2011; 43: E85

(c) Georg Thieme Verlag KG Stuttgart · New York . ISSN 0013-726X

Corresponding author

Z.-Z. Ge, MD

Department of Gastroenterology

Shanghai Renji Hospital

Shanghai Jiaotong University School of Medicine

Shanghai Institute of Digestive Disease

145 Shan-dong zhong Rd

Shanghai 200001

China

Fax: +86-21-68383015

zhizhengge@yahoo.com.cn 